\title{
Estado Funcional De Los Pacientes Con Trauma Craneo Encefalico Criticamente Enfermos
}

\author{
${ }^{1}$ Maria A Rubio, ${ }^{2}$ Juan J Rubio, ${ }^{3}$ Abner Lozano, ${ }^{4}$ Jaime Ruiz, ${ }^{5}$ Juan Sanjuan, ${ }^{6}$ Wilmer F Botache
}

\section{RESUMEN}

Antecedentes: El trauma craneoencefálico (TCE) continúa siendo la principal causa de morbimortalidad entre pacientes con trauma, la discapacidad y el compromiso cognitivo corresponde a la principal afectación configurando pronóstico a largo plazo.

Materiales y métodos: Se realizó un estudio prospectivo tipo cohortes/casos y controles donde se incluyeron los pacientes con TCE severo (Escala Coma de Glasgow <8 y/o TAC anormal) que ingresaron a la unidad de cuidados intensivos Adultos durante 6 meses; se buscó determinar los desenlaces relacionados con el TCE en términos de mortalidad y discapacidad.

Resultados: Se encontraron 32 pacientes, más del 90\% hombres, la mayoría con estabilidad hemodinámica al ingreso, se practicó neurocirugía en el $40 \%$ de los pacientes, aunque todos tenían indicación de monitoria de presión intracraneana solo se realizó en el $6 \%$ de los pacientes. El puntaje en la escala de desenlaces de Glasgow presento una mediana de 3 ( $R I C=225$ puntos). Se clasifico la severidad de la discapacidad en buena recuperacion $9,3 \%$, discapacidad moderada $31,25 \%$, discapacidad severa-muerte hasta en un $25 \%$. Se determino que que un puntaje en la ECG se relacionaba con un GOS mas bajo; la neumonía aspirativa fue la principal complicación extracraneal, la mortalidad global correspondió a un $34.4 \%$.

Conclusión: EI TCE continua afectando de manera especial a las poblaciones jóvenes, con una alta incidencia de consecuencias discapacitantes y alta mortalidad, los centros de trauma deben implementar protocolos mas estrictos para promover la prevención y reforzar la aplicación de recomendaciones mundiales para mejores resultados en términos supervivencia y calidad de vida.

Significancia clinica: El trauma craneoencefálico, continua configurándose como la principal causa de morbimortalidad y discapacidad en adultos jovenes, con esta investigación se busca conocer y describir los datos epidemiológicos relevantes

\footnotetext{
${ }^{1}$ Medica General, ${ }^{2}$ Medico Interno, ${ }^{3}$ Coordinador UCI Adultos ${ }^{4}$ Medico Internista Intensivista, ${ }^{5}$ Residente de Cirugia General ${ }^{6}$ Cirujano de Trauma y Emergencias

1,2,5,6 Departamento de Cirugía General, Universidad Surcolombiana, Neiva, Huila, Colombia

${ }^{3,4}$ Unidad De Cuidados Intensivos, Hospital Hernando Moncaleano Perdomo, Neiva, Huila, Colombia

Corresponding Author: Maria A Rubio, Medica General Departamento de Cirugía General, Universidad Surcolombiana Neiva, Huila, Colombia, teléfono: +5782681997 , e-mail: alerubi3@gmail.com
}

con respecto al desenlace de dicho trauma, conocimiento que es clave en el proceso de enseñanza buscando ofrecer mejores y mas efectivos abordajes en el momento de verse enfrentado a un trauma craneoencefálico.

Palabras claves: Discapacidad, El trauma craneoencefálico, Escala pronostica de Glasgow, Monitoria de pic, Unidad de cuidados intensivos.

\section{ABSTRACT}

Introduction: Traumatic brain injury (TBI) remains to be the leading cause of morbidity and mortality among patients with trauma, disability and neurocognitive commitment corresponds to the main affectation and set the long-term prognosis of these patients.

Materials and methods: It was performed a prospective cohort/cases and controls study, where all the patients with severe TBI (Glasgow Coma Scale (GCS) < 8 and/or abnormal CT scan) were admitted into the adult intensive care unit (ICU) for 6 months to determine the outcomes related to TBI in terms of mortality and disability.

Results: Thirty-two patients, more than $90 \%$ men, most with hemodynamic stability at admission; neurosurgery was practiced in $40 \%$ of patients. Although all had indication of intracranial pressure (ICP), monitoring was only performed in $6 \%$ of the patients. The score on the Glasgow outcome scale presented a median of 3 (IQR $=2 \mathrm{p} 5$ points). The severity of disability in good recovery was $9.3 \%$, moderate disability $31.25 \%$, and severe-dead disability was up to $25 \%$. It was determined that a GCS score was associated with a lower GOS; aspiration pneumonia as the main extracranial complication was presented, overall mortality fell to $34.4 \%$ with only $12.5 \%$ of organ donors.

Conclusion: The TBI continues to affect especially young populations with a high incidence of disabling consequences and high mortality. The trauma centers should implement more strict protocols to promote prevention and to strengthen the implementation of global recommendations for better results in terms of survival and quality of life.

Clinical Significance: Traumatic brain injury continues being the main cause of morbidity and disability in young adults. This research seeks to know and describes the relevant epidemiological data regarding the outcome of this trauma, a knowledge that is key in the teaching process seeking better and more effective approaches at the moment of approach traumatic brain injury.

Keywords: Disability, Glasgow outcome scale, Intensive care unit, Intracranial pressure, Traumatic brain injury.

How to cite this article: Rubio MA, Rubio JJ, Lozano A, Ruiz J, Sanjuan J, Botache WF. Estado Funcional De Los Pacientes

Second place award winner in student category in PTS Congress, Maceio, Brazil, November 10, 2016. 
Con Trauma Craneo Encefalico Criticamente Enfermos. Panam J Trauma Crit Care Emerg Surg 2017;6(3):177-181.

Source of support: Nil

Conflict of interest: None

\section{INTRODUCCION}

El TCE continúa siendo la principal causa de morbimortalidad entre los pacientes con trauma, ${ }^{1}$ de acuerdo con el Center for Disease Control and Prevention (CDC) hasta 1.7 millones sufren TCE, 530000 mueren y hasta 500000 sufren daño neurológico permanente. ${ }^{2}$

Las campañas de prevención buscan limitar la exposición y la aparición de lesiones primarias en pacientes sanos, las lesiones secundarias están bajo el manejo del personal asistencial, siendo la hipertensión intracraneana (HTIC) una de las principales complicaciones y teniendo en cuenta, que de cuya evolución dependen los resultados inmediatos y el pronóstico neurológico de los pacientes, es de vital importancia prestar el manejo más adecuado para los resultados más favorables.

Según la doctrina de Monroe and $\mathrm{Kellie}^{3}$ la suma de los 3 componentes intracraneales (cerebro, volumen sanguíneo y líquido cefalorraquídeo) es constante, cualquier incremento en uno de ellos será a expensas de la disminución de otro como compensación; y en consecuencia, la elevación de la $\mathrm{PIC}^{4}$ como en el caso del trauma craneoencefálico severo y HTIC secundaria. Los valores normales de PIC se describen en la tabla 1.

La HTIC como lesión secundaria más frecuente en el TCE corresponde a la complicación más temida, añadiendo un alto porcentaje de morbimortalidad y desenlaces deletéreos en el paciente, luego, el control de la presión intracraneana (PIC) es uno de los pilares más importantes dentro del tratamiento integral del paciente con lesiones cerebrales graves, además, se convierte en el principal factor de riesgo modificable ${ }^{5}$ del que depende una gran influencia en el resultado final de los pacientes.

Los desenlaces en el trauma craneoencefálico están representados en las etapas iniciales a través de la mortalidad global y el grado de discapacidad; ${ }^{6}$ aunque dichos desenlaces, corresponden a largo plazo, a un gran espectro de entidades y complicaciones manifestados en la vida diaria.

Tabla 1: Valores máximos aceptados de PIC antes de iniciar manejo ${ }^{15}$

\begin{tabular}{ll}
\hline Grupo/Comorbilidad & Valor máximo PIC \\
\hline Adulto sano en decúbito & $15 \mathrm{~mm} \mathrm{Hg}$ \\
Niño & $7 \mathrm{~mm} \mathrm{Hg}$ \\
TCE adulto & $20 \mathrm{~mm} \mathrm{Hg}$ \\
TCE niño & $15 \mathrm{~mm} \mathrm{Hg}$ \\
Cráneo Abierto & $15 \mathrm{~mm} \mathrm{Hg}$ \\
\hline
\end{tabular}

Existen multiples factores que influyen en el desenlace finales, no solo propios del individuo (genero, edad, comorbilidades $^{7}$ ), sino los que involucran la mecánica del trauma, los sistemas de trauma e incluso el abordaje terapéutico prehospitalario. Se ha descrito inclusive la relación entre el nivel educativo, estado civil y uso de casco como medida protectiva. ${ }^{8}$

Por lo general, la discapacidad se relaciona con déficit neurológico ya sea motor o sensitivo, o, a compromiso cognitivo; cada uno con grados individuales de severidad que definirán pronostico.

Los estudios actuales se enfocan en determinar el alcance del trauma craneoencefálico en términos de afectación y deterioro neurológico, en base a la capacidad de retornar al punto de ser individuos independientes y funcionales en la sociedad; se describen múltiples escalas para hacer dichas aseveraciones, incluso se han creado aplicaciones para acercarse a un pronostico mas acertado según diferentes enfoques. ${ }^{9}$

Teniendo en cuenta la trascendencia del TCE, se realizó este estudio con el objetivo de analizar la experiencia institucional a través de los desenlaces en pacientes con TCE severo atendiendo a las recomendaciones de la TBF, proporcionando estadísticas propias relacionadas con la discapacidad y mortalidad en nuestro medio.

\section{MATERIALES Y METODOS}

Tipo de estudio: Prospectivo tipo cohortes/casos y controles

De acuerdo a la naturaleza de las variables se realizaron las medidas de resumen:

- Para las variables continuas se evaluó la normalidad de la distribución con la prueba de SaphiroWilk, cuando no se rechace la hipótesis de nulidad (Ho = Distribución normal) se utilizó la media y la desviación estándar como medidas de resumen. Cuando se rechazó de la hipótesis de nulidad $(\mathrm{Ha}=$ No corresponde a una distribución normal) se utilizó la mediana y el rango intercuartilico como medidas de resumen.

- Para las variables categóricas: Se resumieron mediante las frecuencias absolutas acompañado de su frecuencia relativa

- Consideraciones clínicas: la severidad de las lesiones y el compromiso de las áreas anatómicas se calificaron de acuerdo a las áreas anatómicas.

- Se evaluaran riesgos mediante un análisis univariado con el cálculo del riesgo relativo (RR), y se analizará la significancia estadística con el cálculo del chi cuadrado o el test exacto de fisher de acuerdo al cumplimiento de los supuestos, el valor de p se considera significativo con un valor menor de 0.05, Intervalo de confianza al 95\% y error alfa 5\%. 
Estado Funcional De Los Pacientes Con Trauma Craneo Encefalico Criticamente Enfermos

Tabla 2: Caracteristicas clinicas al ingreso en el servicio de urgencias

\begin{tabular}{ll}
\hline & Med (RIC) \\
\hline Signos Vitales & \\
Cardiaca (Ipm) & $87(74-109)$ \\
Respiratoria (rpm) & $20(17-23)$ \\
TAS (mmHg) & $127(114-144)$ \\
ECG & \\
Motor & $3(2-4)$ \\
Verbal & $1(1-2)$ \\
Ocular & $1(1-2)$ \\
\hline TAS: Tension arterial sitolica: ECG: Escala de Coma de Glasgow
\end{tabular}

TAS: Tension arterial sitolica; ECG: Escala de Coma de Glasgow

\section{Diseño del Estudio}

Análisis descriptivo de subgrupo de una cohorte prospectiva de pacientes con trauma craneonecefalico. Se analizaron los desenlaces obtenidos de los pacientes con TCE severo críticamente enfermos; los datos del estudio incluyeron variables sociodemográficas, el Injury Severity Score (ISS), Trauma Score Revisado (RTS), el abreviado de lesiones de gravedad (AIS) de las lesiones concomitantes, el puntaje de la Escala Coma de Glasgow (ECG), la escala pronostica de Glasgow (GOS), clasificación de los hallazgos tomograficos según el Trauma Coma Data Bank (TCDB), signos vitales, el TCE fue clasificado como el hematoma epidural (EDH), subdural Hematoma (SDH), hematoma intraparenquimatoso (IPH), hemorragia subaracnoidea (HSA), contusiones, edema, herniación, cisternas de la base comprimidas y fracturas de cráneo. La medida de resultado primario fue el estado funcional y la mortalidad hospitalaria. Los resultados secundarios incluyeron complicaciones, duración de la estancia en UCI y otros tratamientos quirúrgicos.

Los criterios de inclusión se basaron en las indicaciones de la guía de la TBF para TCE severo:

- Edad $>18$ años

- ECG $<8$ y/o TAC cerebral anormal (hematomas subdurales/epidural/intraparenquimatoso, hemorragia subaracnoidea, contusiones, edema, herniación, cisternas de la base comprimidas o fracturas de cráneo)

- ECG > 8 y TAC cerebral anormal (hematomas subdurales/epidural/intraparenquimatoso, hemorragia subaracnoidea, contusiones, edema, herniación, cisternas de la base comprimidas o fracturas de cráneo)

- ECG $<8$, TAC normal y al menos 2 de las siguientes: $>40$ años, $\mathrm{PAS}<90 \mathrm{~mm} \mathrm{Hg}$ y postura anormal unilateral o bilateral.

\section{Consideraciones Eticas}

Se realizó un estudio de tipo prospectivo de carácter descriptivo, no se realizó ningún tipo de intervención, se utilizó como fuente primaria las historias clínicas, previo consentimiento del comité de ética
Tabla 3: Lesiones descritas del trauma cráneo encefálico

\begin{tabular}{ll}
\hline Hallazgos & $n(\%)$ \\
\hline Hemorragia Subaracnoidea & $24(75.0)$ \\
Hematoma Subdural & $9(28.1)$ \\
Hematoma Epidural & $7(21.9)$ \\
Hematoma Intraparenquimatoso & $6(18.8)$ \\
Contusion & $16(50.0)$ \\
Fractura craneo & $23(71.9)$ \\
Herniacion & $2(6.3)$ \\
Edema cerebral & $18(56.3)$ \\
Neumoencefalo & $3(9.4)$ \\
\hline
\end{tabular}

del Hospital Universitario Hernando Moncaleano Perdomo. Clasificación de riesgo según la ley colombiana: Investigación con riesgo minimo (Resolución 8430 de 1993). No se tiene ningún conflicto de interés.

\section{RESULTADOS}

Se encontraron un total de 32 pacientes, con una mediana de edad de 25 años y 29 (90.6\%) de ellos eran hombres. la mediana de estancia fue de 20 dias (RIC $=8$ S32 dias). Las medianas de la medida de signos vitales al ingreso presentaron una tendencia a encontrarse dentro de rangos normales (Ver tabla 2). Tres pacientes $(9.4 \%)$ se encontraban en situación de paro cardio cerebro respiratorio.

Después de la atención inicial del servicio de urgencias 18 pacientes $(56.3 \%)$ fueron admitidos a la unidad de cuidado intensivo y 13 pacientes $(40.6 \%)$ fueron llevados a cirugía, las lesiones intracraneales encontradas se encuentran descritas en la Tabla 3. Las lesiones concomitantes al trauma cráneo encefálico fueron tórax en 6 pacientes $(18.8 \%)$ y un paciente presento trauma abdominal. Todas las lesiones del trauma cráneo encefálico fueron calificados como severas [AIS =5]. Aunque todos los pacientes tenían indicación de uso de ventriculostomia para monitoria de PIC solo se realizó en 2 de ellos (6,3\%); los procedimientos realizados se detallan en la Tabla 4 .

Las complicaciones más frecuentemente observadas fue la neumonía aspirativa en 13 pacientes $(40.6 \%)$, aunque también se presentó cerebro perdedor de sal en un $34 \%$ y diabetes insípida en un $22 \%$.

Tabla 4: Procedimientos para manejo del aumento de la presión intra craneal en pacientes con trauma cráneo encefálico

\begin{tabular}{ll}
\hline Procedimientos & $n(\%)$ \\
\hline Drenaje hematoma intraparenquimatoso & $1(3.1)$ \\
Craniectomia descompresiva & $7(21.9)$ \\
Drenaje de hematoma subdural & $8(25.0)$ \\
Drenaje de hematoma epidural & $8(25.0)$ \\
Lobectomia parcial & $1(3.1)$ \\
Esquirlectomia & $3(9.4)$ \\
Drenaje hematoma intraparenquimatoso & $1(3.1)$ \\
\hline
\end{tabular}




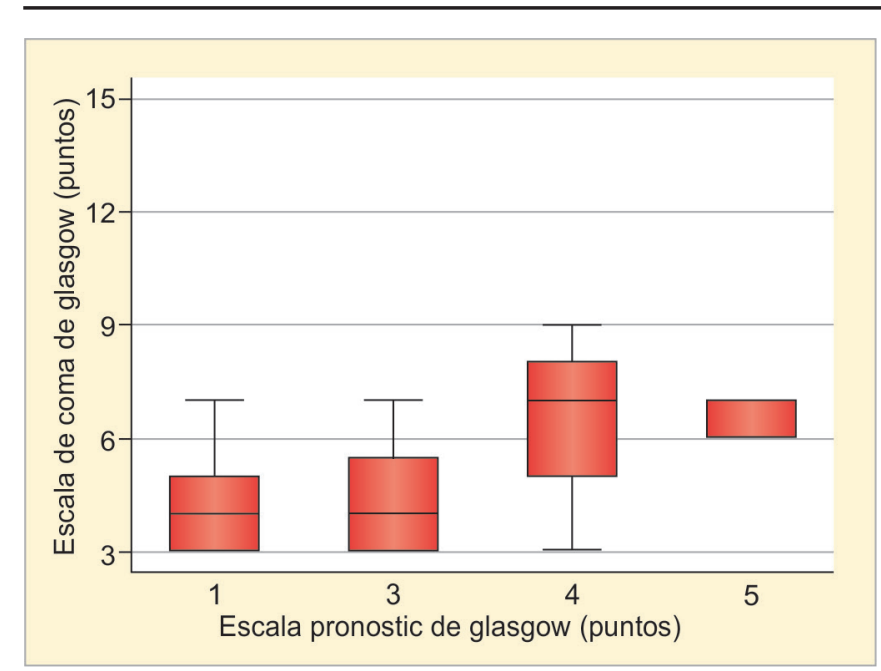

Grafico 1: Asociación ECG de ingreso y GOS

El puntaje en la escala de desenlaces de Glasgow presento una mediana de 3 (RIC $=225$ puntos). Se clasifico la severidad de la discapacidad en buena recuperacion $9,3 \%$, discapacidad moderada $31,25 \%$, discapacidad severa hasta en un $25 \%$.

Se logro determinar que aquellos pacientes que ingresaron con un puntaje en la ECG menor fueron aquellos que obtuvieron un GOS mas bajo, es decir, con un desenlace desfavorable, la relación entre la ECG al inngreso con GOS se encuentra represenntada en la Grafico 1.

Con respecto a la TCDB (Trauma Coma Data Bank) se encontró 4 pacientes incluidos en la Clasificacion Difusa I, 10 pacientes en Difusa II, de los cuales un $40 \%$ se llevo a neurocirugía (sin tener indicación) y una mortalidad del 10\% (sin neurocirugía), 15 pacientes en Difusa III de los cuales un $46.6 \%$ (7 pacientes) fueron llevados a cirugía (aunque todos tenian indicación) y una mortalidad del $46.6 \%$ de los cuales se practico neurocirugía solo en 4 pacientes; en Difusa IV se encontraron 3 pacientes a todos se les realizo neurocirugía con una mortalidad del $100 \%$.

La mortalidad global observada fue del $34.4 \%$ (11 pacientes), de los cuales solo 4 pacientes fueron donantes de órganos. Respecto a la intervencion quirurgica no se encontraron diferencias estadisticamente significativas, con una tendencia a un mayor riesgo de 1.75 veces (sin ajustes) de morir en los pacientes que fueron llevados a una intervencion quirúrgica $[R R=1.75$ (IC 95\% $=0.68=4.55) \mathrm{p}=0.2459]$

\section{DISCUSION}

Epidemiológicamente los datos relacionados con trauma general apuntan a una población muy específica que incluye individuos jóvenes de genero masculino, nuestro estudio siguió la tendencia de la literatura universal, reportando hombres en su mayoría jóvenes afectados por
TCE, datos que concuerdan con estudios como Dahm y cols., ${ }^{10}$ Agrawal y cols. ${ }^{11}$ entre otros; Esta predisposición en la población joven tiene una gran compromiso social, las implicaciones económicas y laborales representadas en años de vida potencialmente perdidos es la mayor motivación para generar políticas de prevención y concientización en la sociedad.

Los grados de discapacidad severa-moderada-buena recuperacion encontrados en el estudio no estuvieron acordes a publicaciones contemporáneas, según Jourdan y cols. ${ }^{6}$ la discapacidad relacionado con el TCE se presento un $32 \%$ severa-muerte, $40 \%$ moderada y un $28 \%$ de buena recuperación comparada con 59,38\%, 31,25\% y $9,3 \%$ respectivamente en nuestro estudio. Es decir, los buenos resultados fueron minimos y los peores desenlaces incluyendo muerte fueron casi el doble con respecto a la literatura; teniendo en cuenta los protocolos establecidos y las indicaciones quirúrgicas en términos de trauma, se puede inferir que mas alla de la severidad del trauma primario se deben complemetar las opciones terapéuticas ofrecidas, siguiendo las recomendaciones mundiales para promover mejores resultados.

Las complicaciones extracraneales que comprometen la función pulmonar (neumonía-SDRA) son la principal comorbilidad relacionada y predisponen a estancias mas prolongadas y a un mayor riesgo de mortalidad, En nuestra estudio encontramos la neumonía como la principal complicación, aunque no se compara a la alta incidencia de otras series como Jovanovic y cols ${ }^{12}$ quienes reportan incidencia de neumonía hasta en la mitad de sus pacientes, Kesinger y $\operatorname{cols}^{13}$ describen la neumonía hasta en un $30 \%$ además de ubicarlo como factor independiente de pronostico a 5 años. Luego, la importancia de la prevención de complicaciones extracraneales influye considerablemente en términos de mortalidad y debe considerarse un pilar importante en el tratamiento integral del paciente con TCE, la predisposición per se de estos pacientes a adquirir infecciones los hacen motivo de cuidados especiales.

El TCE es una entidad altamente mortal, aunque la mortalidad es variable, se encontró una mortalidad menor comparada con otros estudios (34.4\%), según Khajavikhan y cols. ${ }^{14}$ su serie reporta una mortalidad de $46.2 \%$ y según la publicación de Baum y cols. ${ }^{15}$ la mortalidad varía entre un $40166 \%$. Se puede determinar que aunque la mortalidad no es comparable con otras series, la severidad en la discapacidad es la que genera el mayor compromiso entre los sobrevivientes y, a través de estos dos indicadores se puede ver el impacto que representa el resultado conjunto terapeutico que va desde una atención prehospitalaria hasta la aplicación de recomendaciones internacionales para manejo de TCE y el respectivo punto de comparación para nuevos protocolos. 


\section{CONCLUSION}

En conclusión, el Trauma Craneoencefalico, es una entidad con una alta morbimortalidad, representa en gran medida la causa primaria de discapacidad severa en términos de trauma y por consiguiente se le atribuye un importante impacto social; se deben generar nuevas y mejores medidas terapeuticas para ofrecer resultados mas sobresalientes.

\section{SIGNIFICANCIA CLINICA}

El trauma craneoencefálico, continua configurándose como la principal causa de morbimortalidad y discapacidad en adultos jovenes, con esta investigación se busca conocer y describir los datos epidemiológicos relevantes con respecto al desenlace de dicho trauma, conocimiento que es clave en el proceso de enseñanza buscando ofrecer mejores y mas efectivos abordajes en el momento de verse enfrentado a un trauma craneoencefálico.

\section{REFERENCIAS}

1. Tang A, Pandit V, Fennell V, Jones T, Joseph B, O'Keeffe T, Friese RS, Rhee P. Intracranial pressure monitor in patients with traumatic brain injury. J Surg Res 2015 Apr;194(2): 565-570.

2. Centers for Disease Control and Prevention. US Department of Health and Human Services. Atlanta, USA: Traumatic Brain Injury \& Concussion. April 17, 2016 Disponible desde: Available from: http://www.cdc.gov/traumaticbraininjury/ statistics.html.

3. Perez-Barcena J, Llompart-Pou JA, O’Phelan KH. Intracranial pressure monitoring and management of intracranial hypertension. Crit Care Clin 2014 Oct;30(4):735-750.

4. Wykes V, Vindlacheruvu R. Intracranial pressure, cerebral blood flow and brain oedema. Surgery (Oxford) 2015 Aug;33(8):355-362.

5. Dawes AJ, Sacks GD, Cryer HG, Gruen JP, Preston C, Gorospe D, Cohen M, McArthur DL, Russell MM, Maggard-Gibbons M, et al. Intracranial pressure monitoring on mortality following severe traumatic brain injury. J Trauma Acute Care Surg 2015 Mar;78(3):492-501.

6. Jourdan C, Bayen E, Pradat-Diehl P, Ghout I, Darnoux E, Azerad S, Vallat-Azouvi C, Charanton J, Aegerter P, Ruet A, et al. A comprehensive picture of 4-year outcome of severe brain injuries. Results from the PariS-TBI study. Ann Phys Rehabil Med 2016 Apr;59(2):100-106.

7. Ozyurt E, Goksu E, Cengiz M, Yilmaz M, Ramazanoglu A. Retrospective analysis of prognostic factors of severe traumatic brain injury in a university hospital in Turkey. Turk Neurosurg 2015;25(6)877-882.

8. Dagher JH, Costa C, Lamoureux J, de Guise E, Feyz M. Comparative outcomes of traumatic brain injury from biking accidents with or without helmet use. Can J Neurol Sci 2016 Jan;43(1):56-64.

9. Iorio-Morin C, Fortin D, Blanchard J. TBI prognosis calculator: a mobile application to estimate mortality and morbidity following traumatic brain injury. Clinl Neurol Neurosurg 2016 Mar;142:48-53.

10. Dahm J, Ponsford J. Comparison of long-term outcomes following traumatic injury: what is the unique experience for those with brain injury compared with orthopaedic injury? Injury 2015 Jan;46(1):142-149.

11. Agrawal D, Ahmed S, Khan S, Gupta D, Sinha S, Satyarthee GD. Outcome in 2068 patients of head injury: Experience at a level 1 trauma centre in India. Asian J Neurosurg 2016 Apr-Jun;11(2):143-145.

12. Jovanovic B, Milan Z, Markovic-Denic L, Djuric O, Radinovic K, Doklestic K, Velickovic J, Ivancevic N, Gregoric P, Pandurovic $\mathrm{M}$, et al. Risk factors for ventilator-associated pneumonia in patients with severe traumatic brain injury in a Serbian trauma centre. Int J Infect Dis 2015 Sep;38:46-51.

13. Kesinger MR, Kumar RG, Wagner AK, Puyana JC, Peitzman AP, Billiar TR, Sperry JL. Hospital-acquired pneumonia is an independent predictor of poor global outcome in severe traumatic brain injury up to 5 years after discharge. J Trauma Acute Care Surg 2015 Feb;78(2):396-402.

14. Khajavikhan J, Vasigh A, Khani A, Jaafarpour M, Kokhazade T. Outcome and predicting factor following severe traumatic brain injury: a retrospective cross-sectional study. J Clin Diagn Res 2016 Feb;10(2):16-19.

15. Baum J, Entezami P, Shah K, Medhkour A. Predictors of outcomes in traumatic brain injury. World Neurosurg 2016 Jun; 90:525-529. 